\title{
P160: The enablers and barriers to introducing "bare below the elbows" for hand hygiene behaviors: an exploratory study
}

\author{
K McKay $^{1 *}$, R Shaban ${ }^{2}$, E Coyne $^{3}$ \\ From 2nd International Conference on Prevention and Infection Control (ICPIC 2013) \\ Geneva, Switzerland. 25-28 June 2013
}

\begin{abstract}
Introduction
Introduction of the 'bare below the elbows' (BBE) guidelines within the National Health Service in the United Kingdom was met with complaints from many health care workers $(\mathrm{HCW})$. BBE has been introduced in several states of Australia, and is expected to become mandatory in Victoria. There is no research indentifying the barriers and enablers to BBE nor any publicly available information on successful implementation programs. This study examines the barriers and enablers to the introduction of $\mathrm{BBE}$ within the 3 largest hospitals of the Eastern Health (EH) Network in outer Melbourne, Australia.
\end{abstract}

\section{Objectives}

The aims of this study were to determine the extent to which the dress and adornment behaviours of HCW at $\mathrm{EH}$ were already consistent with the principles of $\mathrm{BBE}$, and to determine the enablers and barriers to the introduction of BBE within clinical areas at $\mathrm{EH}$.

\section{Methods}

The study was descriptive and utilized both quantitative and qualitative methodology. Data was collected at the 3 major EH sites in 2 phases, a point prevalence audit which described the current hand adornment and HCW dress behaviours as compared to the BBE framework and focus groups to explore $\mathrm{HCW}$ opinions and feelings surrounding the changes inherent in $\mathrm{BBE}$.

\section{Results}

The audit showed that overall $11.7 \%$ of staff were compliant with BBE. Data was also examined according to

${ }^{1}$ Infection Prevention \& Control, Eastern Health, Box Hill, Brisbane, Australia Full list of author information is available at the end of the article site, gender, ward type, HCW group and BBE element. A picture of the dress and hand adornment practices of the target staff was thus able to be quantified. Barriers and enablers include the lack of a uniform, heating, clocks, pass holders and storage. In addition broader issues such as consequences, feedback, evidence, equity, identity, role modelling and organizational support were also identified.

\section{Conclusion}

Achieving compliance with BBE is possible but would require consideration of multiple factors such as those illuminated by this work; medical staff may prove a difficult challenge with regard to sleeve length and the wearing of ties, while the removal of rings is an emotive area. It is anticipated that this data will allow the formulation of strategies to introduce these practice changes in an efficient, cost effective and sustainable way which does not adversely affect other infection prevention strategies within $\mathrm{EH}$.

\section{Disclosure of interest}

None declared.

\section{Author details}

'Infection Prevention \& Control, Eastern Health, Box Hill, Brisbane, Australia. ${ }^{2}$ Griffith Health Institute, Brisbane, Australia. ${ }^{3}$ School of Nursing, Griffith University, Brisbane, Australia.

Published: 20 June 2013

doi:10.1186/2047-2994-2-S1-P160

Cite this article as: McKay et al.: P160: The enablers and barriers to introducing "bare below the elbows" for hand hygiene behaviors: an exploratory study. Antimicrobial Resistance and Infection Control 2013 2(Suppl 1):P160.

\section{C)

